\title{
Kernos
}

Revue internationale et pluridisciplinaire de religion grecque antique

$26 \mid 2013$

Varia

\section{Nadine Deshours, L'été indien de la religion civique. Étude sur les cultes civiques dans le monde égéen à l'époque hellénistique tardive}

\section{Stéphanie Paul}

\section{OpenEdition}

1 Journals

\section{Édition électronique}

URL : http://journals.openedition.org/kernos/2159

DOI : 10.4000/kernos.2159

ISSN : 2034-7871

\section{Éditeur}

Centre international d'étude de la religion grecque antique

\section{Édition imprimée}

Date de publication : 10 octobre 2013

Pagination : 406-410

ISSN : 0776-3824

\section{Référence électronique}

Stéphanie Paul, « Nadine Deshours, L'été indien de la religion civique. Étude sur les cultes civiques dans le monde égéen à l'époque hellénistique tardive », Kernos [En ligne], 26 | 2013, mis en ligne le 10 octobre 2013, consulté le 02 mars 2021. URL : http://journals.openedition.org/kernos/2159 ; DOI : https://doi.org/10.4000/kernos.2159

Ce document a été généré automatiquement le 2 mars 2021.

Kernos 


\title{
Nadine Deshours, L'été indien de la religion civique. Étude sur les cultes civiques dans le monde égéen à l'époque hellénistique tardive
}

\author{
Stéphanie Paul
}

\section{RÉFÉRENCE}

Nadine Deshours, L'été indien de la religion civique. Étude sur les cultes civiques dans le monde égéen à l'époque hellénistique tardive, Bordeaux, Ausonius, 2011.1 vol. 19,5×24 cm, 408 p. (Scripta Antiqua, 30). ISBN : 978-2-35613-037-2.

1 La religion hellénistique a été longtemps perçue, à tort, comme un déclin de la religion des périodes antérieures. Cette perception, souvent fondée sur la généralisation abusive de cas isolés, définissait comme caractéristiques principales de cette période une individualisation de la démarche religeuse, l'émergence d'une piété plus personnelle et une plus grande popularité des cultes étrangers, dits «orientaux » ou égyptiens, aux dépens des cultes traditionnels de la polis. Cette perspective était due à une large méconnaissance des dossiers documentaires de cette période, enclavée entre deux autres périodes considérées plus prolifiques, la période classique et l'Empire romain. Plus récemment, un intérêt grandissant pour la polis de l'époque hellénistique a conduit les chercheurs à remettre en question l'évolution de la religion telle qu'elle était autrefois présentée et à réévaluer les changements et continuités caractéristiques de l'époque ${ }^{1}$.

2 C'est donc le grand mérite de l'ouvrage ici recensé d'aborder la problématique de la religion à l'époque hellénistique en se fondant sur une analyse étroite de dossiers documentaires, en l'occurrence les documents officiels de la polis. Se concentrant plus particulièrement sur la période hellénistique tardive, à savoir les $\mathrm{II}^{\mathrm{e}}$ et $\mathrm{I}^{\mathrm{er}} \mathrm{s}$. avant notre 
ère, l'A. entend dresser un portrait de la «religion civique ", définie comme « système de cultes financé, organisé, contrôlé par la cité parce qu'il en fonde et maintient l'identité "(p. 303). L'un des arguments principaux est également de savoir si "l'exception» athénienne en est vraiment une, à savoir si les caractéristiques de la religion d'Athènes, qui jouissait à cette période d'une grande prospérité en raison de sa situation politique particulière, sont applicables au monde égéen en général.

3 Après avoir exposé la problématique de l'ouvrage dans une présentation et une introduction, la première partie se concentre sur la question de la périodisation. Dans le sillage des travaux de Philippe Gauthier, l'A. remet en question la cohérence et l'unité d'une période hellénistique envisagée d'un seul bloc. Elle produit donc un inventaire des documents officiels touchant à la religion civique depuis le milieu du IV ${ }^{\mathrm{e}}$ s. jusqu'au début de l'époque impériale - à l'exclusion des cultes royaux - et classés selon trois principes différents: chronologique, géographique et typologique. Ce panorama lui permet de confirmer la distinction de Ph. Gauthier entre la haute époque hellénistique ( $\mathrm{IV}^{\mathrm{e}}-\mathrm{II}^{\mathrm{e}} \mathrm{s}$.) et l'époque hellénistique tardive ( $\mathrm{II}^{\mathrm{e}}-\mathrm{I}^{\mathrm{er}} \mathrm{s}$.), qui retiendra son attention dans la suite de l'ouvrage. Ce sont pourtant trois périodes-clés qui concentrent les « réformes ou restauration de cultes » (la fin du IV ${ }^{\mathrm{e}}$ s.; le tournant des $\mathrm{III}^{\mathrm{e}}$ et $\mathrm{II}^{\mathrm{e}} \mathrm{s}$.; le tournant des $\mathrm{II}^{\mathrm{e}}$ et $\mathrm{I}^{\mathrm{er}} \mathrm{s}$.) et trois phases différentes dans les tendances religieuses qu'elle identifie : 1) la création de fêtes à la haute époque hellénistique; 2) la "panhellénisation" des fêtes au $\mathrm{III}^{\mathrm{e}}$ s.; 3) un mouvement de restauration ou de "solennisation" des cultes et l'importance de fondations privées pour des cultes civiques à l'époque hellénistique tardive. Un tel inventaire des sources épigraphiques pourra sans nul doute s'avérer utile pour quiconque s'intéresse au sujet, même si la forme des tableaux donne une impression quelque peu confuse. Peut-être un développement de la problématique en introduction renvoyant à une simple annexe avec la liste des documents eût-il été préférable. On notera çà et là quelques imprécisions, notamment sur la nature des documents. Par exemple, ce qui est dénommé le « règlement cultuel » de Nikè à $\operatorname{Cos}(\mathrm{p} .45)$ est en fait une vente de prêtrise. Dès lors, pourquoi prendre cette inscription en considération et non les dizaines d'autres documents similaires en provenance de l'Égée et de l'Asie mineure, puisqu'il s'agit bien de documents émanant de la polis et réglementant des cultes publics? La datation de certaines inscriptions est occasionnellement problématique. L'inscription de Magnésie du Méandre sur "l'inauguration de la statue " (LSAM 33A : il s'agit en réalité d'une réinstallation, comme il sera exposé plus loin) est datée du début du $\mathrm{II}^{\mathrm{e}} \mathrm{s}$. (p. 43) alors que l'A. adopte plus loin (p. 198) la date de la fin du III $\mathrm{e}$ s. De même en ce qui concerne les Asclepieia de Cos : la construction du temple et l'instauration des fêtes sont placées au début du III $\mathrm{s}$. (p. 37, où n'est citée aucune référence à une inscription, mais un renvoi à la bibliographie), fêtes qui auraient été « réorganisées » en 242 (p. 39), voire à la fin du III s. selon la p. 57 et la p. 205. En réalité, si la monumentalisation du temple est bien datée de la première moitié du $\mathrm{III}^{\mathrm{e}} \mathrm{s}$. par l'archéologie, les Asclepieia sont instaurées en 242 et ont dès le départ un caractère panhellénique ${ }^{2}$.

4 En ce qui concerne les deux autres parties de l'ouvrage, l'A. procède à l'analyse précise de dossiers documentaires consacrés à une thématique particulière. Elle y cite les sources, reproduit les textes importants en grec avec une traduction, ainsi que certains documents utiles (plans et cartes). Le tout est accompagné d'un commentaire exhaustif de l'inscription, mais également d'une remise en contexte systématique. 
5 Dans la deuxième partie, «L'exception athénienne? ", l'A. reprend la documentation traitée dans le chapitre intitulé "Indian Summer » de l'ouvrage Religion in Hellenistic Athens de J.D. Mikalson ${ }^{3}$ - intitulé qui est d'ailleurs à l'origine du titre du présent ouvrage -, mais l'aborde selon une méthode quelque peu différente, examinant les documents au plus près. Depuis la restitution de Délos (167/66) jusqu'à la crise mithridatique (88), Athènes a en effet connu une grande prospérité en raison de l'alliance avec Rome, prospérité qui aurait rejailli sur les cultes en un mouvement de restauration calqué sur le modèle de Lycurgue. Quatre dossiers distincts sont envisagés. Dans le premier, qui concerne la restauration de cultes ou de fêtes abandonnés, l'A. souhaite mettre en évidence la réaffirmation des relations ancestrales entre Athènes et Delphes, avec la réinstauration de la Pythaïde, la réaffirmation des valeurs civiques, via le culte d'Apollon Patrôos, étroitement lié à la définition de la citoyenneté, et celui de Thésée, associé à l'unité de l'Attique et modèle de l'éphébie, et enfin la vitalité des cultes dans les dèmes. Le deuxième dossier concerne la "solennisation" de cultes traditionnels. Il s'agit d'un nouvel éclat donné à ces cultes sans que l'on puisse parler d'une restauration proprement dite puisqu'il n'y a pas d'interruption. La documentation sur laquelle se fonde cet argument est principalement constituée de décrets honorifiques pour les ergastinai, chargées de tisser le péplos d'Athéna, pour le personnel de culte éleusinien, et pour les prêtres d'Asclépios. Invoquer ce type de documentation pour suggérer un « renouveau » des cultes traditionnels n'est toutefois pas sans poser question, car l'accroissement des honneurs rendus aux prêtres pourrait aussi bien relever d'un changement d'ordre social. Les décrets honorifiques pour des prêtres sont d'ailleurs bien attestés durant toute la période hellénistique ${ }^{4}$. Cette partie comprend encore un long développement sur l'éphébie, qui, tout en s'ouvrant aux étrangers, se définit par un retour à la tradition, et un autre, plus bref, sur les relations entre Athènes et Délos. En conclusion, la religion athénienne de cette période se caractérise par un retour aux coutumes ancestrales (patria), qui s'accompagne de quelques innovations sur le plan social, notamment le recours à quelques membres de l'élite pour financer les cultes. Par la mise en place de cette politique cultuelle, Athènes renforcerait son unité et son identité et, sur le plan extérieur, elle s'afficherait comme une exception au sein du monde grec.

6 La troisième partie veut mettre à l'épreuve ce modèle athénien avec la situation des autres cités du monde égéen : cinq thématiques sont mises en évidence et illustrées par plusieurs dossiers. La première concerne la réorganisation des cultes, dont l'A. donne pour exemple le cas de l'Artémis Leukophryènè de Magnésie du Méandre et de l'instauration des Eisitèria à l'occasion de la reconstruction du temple et de la réinstallation du xoanon de la déesse, connus par deux décrets successifs (LSAM 33A et 33B). À cette occasion, les résidents (katoikountes) de Magnésie sont invités à adresser des sacrifices sur des autels particuliers érigés devant leur maison, ce que l'A.interprète, de manière intéressante, comme une manière d'inclure dans la célébration de la déesse tutélaire de la cité des non citoyens. Cette bonne présentation $\mathrm{du}$ dossier souffre toutefois d'une certaine confusion dans la chronologie. Par exemple, après avoir adopté la date de la fin du $\mathrm{III}^{\mathrm{e}} \mathrm{s}$. pour le premier décret et d'après 129 pour le second, l'A. semble souscrire à l'hypothèse de Hiller von Gaërtringen qui considère que l'instauration des Eisitèria aurait été motivée par la guerre d'Aristonicos, en 129 (p. 205). Or, le nom Isitèria est attesté dès le premier décret (1.25), et le second ne fait que remettre en vigueur des mesures prises auparavant et proposer son inscription sur un montant du portique du sanctuaire ${ }^{5}$. Plus loin (p. 207), l'A. semble confondre les 
Eisitèria et les Leukophryènè panhelléniques en parlant d'une « internationalisation » de la fête. Cette thématique de réorganisation des cultes est encore illustrée par le dossier de Coropè, qui comporte deux règlements sur le fonctionnement de l'oracle et la préservation du bois sacré d'Apollon Coropaios. Ces inscriptions trouvent leur origine dans l'affluence des pélerins dans le sanctuaire et attestent la vitalité continue du culte. Par la deuxième thématique (Identités civiques et «légendes nationales »), l'A. veut mettre en évidence le rôle de la religion dans la formation de l'identité de la polis. Sont ainsi invoqués les cas de la Chronique de Lindos, par laquelle une communauté s'est forgée un passé mythique prestigieux en vue d'affirmer son identité, et celui des mystères d'Andanie, qui constitue en quelque sorte un résumé de son livre consacré à la question ${ }^{6}$. Le troisième point (Les élites au secours des cultes civiques) donne plusieurs exemples de fondations privées pour des cultes publics, venant ainsi pallier les difficultés financières que pouvaient éprouver les cités : la mise par écrit de règlements concernant le culte d'Athéna Nikèphoros à Pergame; la fondation d'Hermias à Ilion; le financement des Itônia et la fondation d'Hègèsarétè à Amorgos. L'A. souligne avec raison qu'il ne s'agit en aucun cas d'une privatisation des cultes, puisque l'argent est géré par la cité, qui continue d'assurer en tout point l'organisation de la fête. Ce sont les innovations qui sont au centre de la quatrième thématique (Des initiatives inattendues). Le cas de la réorganisation de la fête en l'honneur d'Artémis Kindyas à Bargylia donne un exemple intéressant de l'intégration des métèques, chargés d'élever des animaux pour le sacrifice, à une célébration civique. Pour expliquer cette participation, qui se déroule toutefois légèrement en marge de celle des citoyens puisqu'il n'y a pas de banquet commun, l'A. donne plusieurs hypothèses : il s'agirait d'imiter les Panathénées d'Athènes, d'intégrer les populations cariennes qui ne font pas partie du corps civique, de renforcer l'unité de la cité ou de reconnaître le rôle joué par les étrangers dans sa prospérité. Le dernier dossier envisagé est celui de Milet et de l'inscription par les Molpes de réglementations concernant les orgia. À ce propos, le lecteur pourra peut-être s'interroger sur la question de savoir dans quelle mesure la réinscription de mesures anciennes, invoquée à quelques reprises dans l'ouvrage, témoigne d'un dynamisme particulier du culte. Enfin, cette troisième partie se conclut par un excursus sur l'évolution du paysage sacré (Désaffection ou regain d'intérêt pour les sanctuaires à l'époque hellénistique tardive?). Ainsi, l'A. conclut à l'encontre de l'idée d'exception athénienne : la religion civique aurait également connu un "été indien » dans d'autres régions du monde grec, fût-ce avec un léger décalage dans le temps.

7 Dans sa conclusion générale, l'A. émet plusieurs remarques intéressantes sur la problématique de la religion à l'époque hellénistique tardive. Notamment, elle met en évidence la tension entre l'idée d'un retour aux coutumes ancestrales (kata ta patria) et un certain nombre d'innovations dues à des évolutions politiques et sociales, telles que le financement privé des cultes par des évergètes, le rôle de diverses associations ou collèges dans l'organisation, le financement ou le déroulement des cultes et la participation des non citoyens aux cultes civiques. Or, le lecteur pourra opposer à cette réflexion que ces caractéristiques ne sont pas typiques de l'époque hellénistique tardive, voire, pour certaines, de la période hellénistique tout court. En effet, l'implication religieuse de divers groupes, notamment familiaux ou civiques, est attestée dès l'époque archaïque, et les Molpes existaient à Milet déjà au $\mathrm{VI}^{\mathrm{e}} \mathrm{s}$. Ces changements relèveraient-ils donc davantage d'une différence de degré ou d'intensité, plutôt que de caractère ? Par ailleurs, la nature civique de la documentation ici prise en considération montre sans conteste qu'on ne peut parler de déclin de la religion à 
l'époque hellénistique. Dans la mesure où les innovations énoncées comme caractéristiques de cette période ont généralement été identifiées non pas au sein de la polis, mais en dehors, dans les dévotions privées et les cultes associatifs, le lecteur pourra alors s'interroger sur le résultat que pourrait donner la confrontation d'une telle étude avec des documents d'autres types, comme ceux qui règlementent les cultes privés, les décrets émis par les associations, les dédicaces, etc. Autant de questions stimulantes que suscite l'ouvrage ici recensé.

Enfin, une légère remarque sur la forme de l'ouvrage, qui n'est pas du fait de l'A., s'impose. En plus du format carré, peu maniable, le renvoi des notes (assez peu fournies) en fin d'exposé, lié au système de citation de références 'auteur + année', est particulièrement peu commode car il multiplie les renvois.

En conclusion, ce livre fournit une bonne présentation de nombre de dossiers épigraphiques, accompagnés d'un commentaire substantiel toujours très ancré dans le contexte historique, mais un commentaire tellement exhaustif qu'il lui arrive d'en perdre de vue l'argument principal. L'ouvrage n'en sera pas moins profitable à tous les historiens de la religion grecque et à ceux qui s'intéressent plus généralement à l'histoire de la période hellénistique.

\section{NOTES}

1. Pour une excellente mise au point récente de la problématique, voir J.D. Mikalson, «Greek Religion: Continuity and Change in the Hellenistic Period ", in G.R. Bugh (éd.), The Cambridge Companion to the Hellenistic World, Cambridge, 2006, p. 208-222.

2. Sur l'Asclépieion de Cos, voir en dernier lieu J.W. Riethmüller, Asklepios: Heiligtümer und Kulte, Heidelberg, 2005 (Studien zu antiken Heiligtümern, 2), vol.1, p. 206-219. Sur l'instauration des Asclepieia, voir le dossier de l'asylie : IG XII 4, 207-245 et les règlements cultuels IG XII 4, 286-287.

3. J.D. Mikalson, Religion in Hellenistic Athens, Berkeley et al., 1998, p. 242-287.

4. Voir à ce propos l'article de S.D. Lambert, «The Social Construction of Priests and Priestesses in Athenian Honorific Decrees from the Fourth Century BC to the Augustan Period», in M. Horster, A. Klöckner (éd.), Civic Priests. Cult Personnel in Athens from the Hellenistic Period to Late Antiquity, Berlin/ Boston, 2012 (Religionsgeschichtliche Versuche und Vorarbeiten, 58), p. 67-133 (recensé dans cette même chronique). Le corpus des décrets honorifiques pour des prêtres d'Asclépios, il est vrai assez exceptionnel par sa taille, pourrait être dû au hasard des fouilles ou au fait que la prêtrise ait été annuelle: voir également dans le même ouvrage E. PerrinSaminadayar, "Prêtres et prêtresses d'Athènes et de Délos à travers les décrets honorifiques athéniens (167-88 a.C.) », p. 135-159.

5. H.v. Gaërtringen date la totalité de l'inscription d'après 129 (Syll. ${ }^{3} 695$ ). Si l'on adopte des dates différentes, il est possible en revanche d'attribuer à la fin de la guerre d'Aristonicos la motivation de réinsister sur ces célébrations à cette période, de même que l'épiclèse Nikèphoros, associée à Artémis dans le second décret uniquement.

6. N. Deshours, Les mystères d'Andanie. Étude d'épigraphie et d'histoire religieuse, Bordeaux, 2006. 


\section{AUTEURS}

\section{STÉPHANIE PAUL}

F.R.S.-FNRS - Université de Liège 